RASĀYAN J. Chem.

Vol. 14 | No. 3 |1534-1538| July - September | 2021 ISSN: 0974-1496 | e-ISSN: 0976-0083 | CODEN: RJCABP http://www.rasayanjournal.com http://www.rasayanjournal.co.in

\title{
SYNTHESIS OF 2-(4-((1-PHENYL-1H-1,2,3-TRIAZOLE-4-YL) METHOXY) PHENYL)QUINAZOLINE-4(3H)-ONE
}

\author{
Thummala Rajamani ${ }^{1}$ and K. Bhaskar ${ }^{2, \bigotimes}$ \\ ${ }^{1}$ Department of Chemistry, University Arts \& Science College, Kakatiya University, Warangal - \\ 506 001, Telangana, India \\ ${ }^{2}$ Department of Chemistry, Osmania University, Hyderabad-500 007, Telangana, India \\ ${ }^{\square}$ Corresponding Author: manithummala6@gmail.com
}

\begin{abstract}
New chemical entities encompassing 1,2,3-triazole and quinazolinones (7a-h) have been synthesized starting from 4-hydroxy benzaldehyde (1) in three steps involving the addition of propoxyl bromide (2), iodine/dimethyl sulfoxide catalyzed condensation of anthranilamide (4) and finally 'Click' reaction with various phenylazides (6a-h). Several analogs have been synthesized in excellent yields and fully characterized by using ${ }^{1} \mathrm{H}$ NMR, IR and Mass spectroscopic methods and they were superior in accordance with the proposed structures.

Keywords: Anthranilamide, 4-hydroxy benzaldehyde, propoxyl bromide, and 1,2,3-triazole substituted quinazolinone.
\end{abstract}

RASĀYAN J. Chem., Vol. 14, No.3, 2021

\section{INTRODUCTION}

The plethora of biological properties, such as anticancer ${ }^{1}$, anti-HIV ${ }^{2}$, antimicrobial ${ }^{3}$ etc, exhibited in the quinazolinone core structure has attracted the immense interest of research groups across the globe. A much often employed strategy to bring about diversity in biologically important core moieties is through the structural modifications employing chemical means. This is especially true in the case of quinazolinones. The literature precedence indicates that the potency of the quinazolinone core structure can be manipulated through suitable structural changes. ${ }^{4-7}$ The structural alterations of a lead compound are a frequently used strategy in drug discovery. Various naturally occurring molecules bearing quinazolinone core have been identified to have important biological properties. Camptothecin, Rutaecarpine, Luotonin etc are some of the examples (Fig.-1). ${ }^{7-15}$

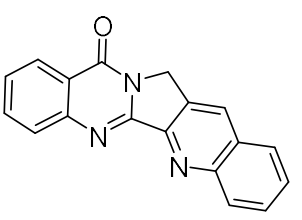

Luotonin A<smiles>CC[C@]1(O)C(=O)OCc2c1cc1n(c2=O)Cc2cc3ccccc3nc2-1</smiles>

Camptothecin<smiles>CCc1nc2ccccc2c(=O)n1-c1c(Cl)cccc1Cl</smiles>

Chloroqualone<smiles>Cc1ccccc1-n1c(CF)nc2ccc(N)cc2c1=O</smiles>

Alloqualone

Fig.-1: Structures of Luotonin A, Camptothecin, Chloroqualone, Alloqualone

In continuation of our ongoing research in search of new biological active compounds, a series of novel analogs of 1,2,3-triazole substituted quinazolinones (7a-h) have been synthesized in the present investigation (Scheme-1).

\section{EXPERIMENTAL}

\section{Material and Methods}

To measure the melting points of the compounds Fischer-Johns melting point apparatus was used. Perkin Elmer-283B \& Nicolet-740 was used to record IR spectra. Varian Gemini-200, Varian unit-500 and Avance $300 \mathrm{MHz}$, Avance $400 \mathrm{MHz}$, BrukerUx-NMR were used to record ${ }^{1} \mathrm{H}$ NMR spectra and VG 
Micro mass $7070 \mathrm{H}$ (ESI was used to record Mass spectra).

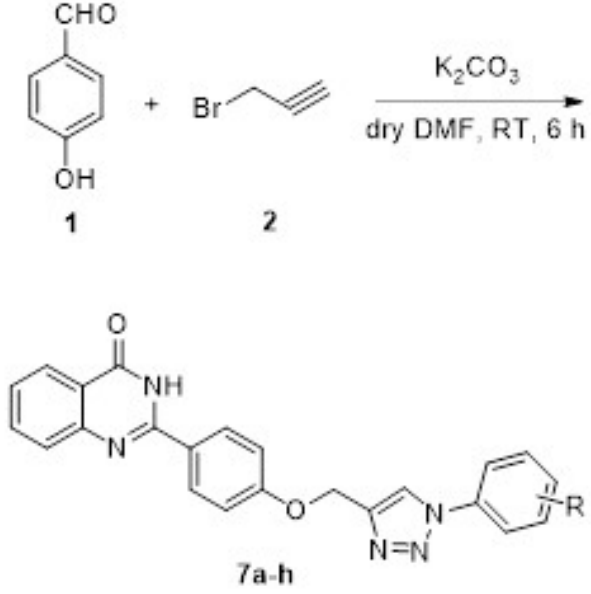

4-(Prop-2-yn-1-yloxy)benzaldehyde (3)
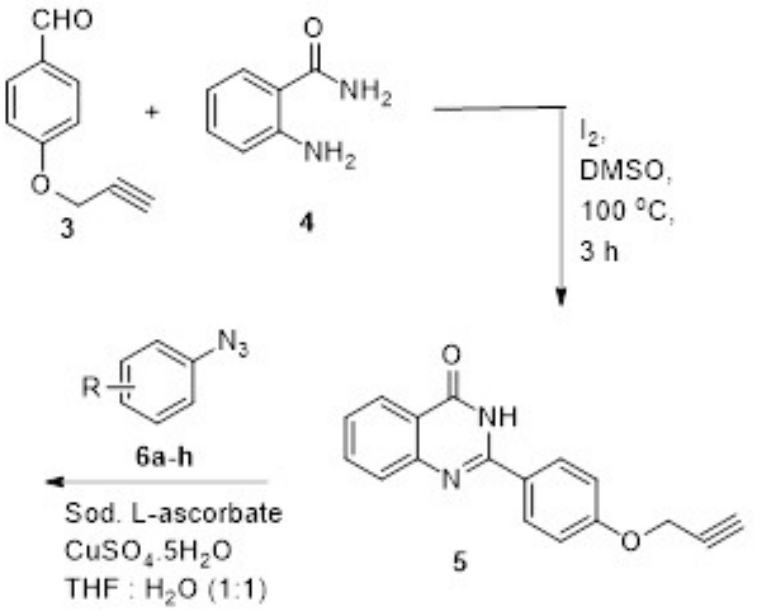

5

Scheme-1

4-Hydroxy benzaldehyde (1, 1 equiv.), propoxyl bromide (2, 1.2 equiv.) was stirred at room temperature with $\mathrm{K}_{2} \mathrm{CO}_{3}$ in dry dimethyl formamide for $6.5 \mathrm{~h}$. The mixture was poured onto crushed ice, formed solid was filtered and dried to get 4-(prop-2-yn-1-yloxy)benzaldehyde (3) as pale yellow. Yield: 90\%, m. p. 64$66{ }^{\circ} \mathrm{C} .{ }^{1} \mathrm{H}$ NMR $\left(\mathrm{CDCl}_{3}\right): \delta 2.59\left(\mathrm{~s}, 1 \mathrm{H}\right.$, alkyne-H), $4.80\left(\mathrm{~s}, 2 \mathrm{H},-\mathrm{OCH}_{2}-\right), 7.10$ (d. $2 \mathrm{H}$, aromatic-H), 7.90 $(2 \mathrm{H}$, aromatic-H), $9.95(\mathrm{~s}, 1 \mathrm{H}$, -aldehyde). ESI-MS: $m / z=161[\mathrm{M}+\mathrm{H}]$.

\section{4-(Prop-2-yn-1-yloxy) phenyl) quinazolin-4(3H)-one (5)}

A mixture of 4-(prop-2-yn-1-yloxy)benzaldehyde (3, 1.3equiv.) and anthranilamide (4, 1equiv.) were heated at $100{ }^{\circ} \mathrm{C}$ for $3 \mathrm{hr}$ in presence of iodine and DMSO. Based on TLC reaction progress tested, the mixture was cooled to RT and hypo solution was used for quenching, then extracted with EtOAc $(3 \times 20$ $\mathrm{mL})$. Ethyl acetate layers were washed with $\mathrm{H}_{2} \mathrm{O}(3 \times 30 \mathrm{~mL})$, dried and concentrated. The pure 4-(prop-2yn-1-yloxy)phenyl) quinazoline-4(3H)-one (5) in excellent yield $(92 \%)$ was obtained upon column chromatography with EtOAc : Hexane (2:8) as eluent; m. p. 166-168 ${ }^{\circ} \mathrm{C} .{ }^{1} \mathrm{H}$ NMR $\left(\mathrm{CDCl}_{3}\right): \delta 3.64(\mathrm{~s}, 1 \mathrm{H}$, alkyne-H), $4.93\left(\mathrm{~s}, 2 \mathrm{H},-\mathrm{OCH}_{2}-\right), 7.14-7.19(\mathrm{~m}, 2 \mathrm{H}$, aromatic-H), 7.48-7.52 (m, 1H, aromatic- $\mathrm{H}), 7.70-$ $7.74(\mathrm{~m}, 1 \mathrm{H}$, aromatic- $\mathrm{H}), 7.80-7.85(\mathrm{~m}, 1 \mathrm{H}$, aromatic- $\mathrm{H}), 8.13-8.17(\mathrm{~m}, 1 \mathrm{H}$, aromatic- $\mathrm{H}), 8.19-8.22(\mathrm{~m}$, $2 \mathrm{H}$, aromatic-H). ESI-MS: $m / z=277[\mathrm{M}+\mathrm{H}]$.

Synthesis of 2-(4-((1-substituted phenyl-1H-1,2,3-triazol-4-yl) methoxy)phenyl)quinazolin-4(3H)one derivatives $(7 \mathbf{a}-\mathrm{h})$

To the mixture of azido compound (6a, 1.4 equiv) and 2-(4-(prop-2-yn-1-yloxy)phenyl) quinazolin-4$(3 H)$-one $\left(5,1\right.$ equiv) in THF: $\mathrm{H}_{2} \mathrm{O}(1: 1)$ was added $\mathrm{CuSO}_{4} \cdot 5 \mathrm{H}_{2} \mathrm{O}(3.2 \mathrm{eq})$ and sodium ascorbate $(3.2 \mathrm{eq})$ at room temperature. The reaction mixture was stirred at RT for 3-4 h. Based on TLC, mixture was extracted with ethyl acetate $(2 \times 10 \mathrm{~mL})$ and water $(5 \mathrm{~mL})$. The organic layer was separated, dried and concentrated to furnish following quinazolin tethered triazoles:

\section{2-(4-((1-Phenyl-1H-1,2,3-triazol-4-yl) methoxy) phenyl)quinazolin-4(3H)-one (7a)}

Yield: $85 \%$, m. p. $232-234{ }^{\circ} \mathrm{C}$. IR (KBr): v 3444, 3058, 1658, 1606, 1564, 1482, $1444 \mathrm{~cm}^{-1} .{ }^{1} \mathrm{H}$ NMR $\left(\mathrm{CDCl}_{3}\right): \delta 5.39\left(\mathrm{~s}, 2 \mathrm{H},-\mathrm{OCH}_{2}\right), 7.15(\mathrm{~d}, J=3.5 \mathrm{~Hz}, 2 \mathrm{H}$, aromatic-H), 7.50-7.62 (m, 2H, aromatic-H), 7.70-7.81 (m, 5H, aromatic-H), 8.14-8.31 (m, 5H, aromatic-H). ESI-MS: $m / z=396[\mathrm{M}+\mathrm{H}]$.

2-(4-((1-(2-(Trifluomethyl)phenyl-1H-1,2,3-triazol-4-yl)methoxy)phenyl)quinazolin-4(3H)-one (7b) Yield: $90 \%$, m. p. $210-212^{0} \mathrm{C}$. IR (KBr): v $3445,3060,1656,1600,1565,1485,1445 \mathrm{~cm}^{-1} .{ }^{1} \mathrm{H}$ NMR $\left(\mathrm{CDCl}_{3}\right): \delta 5.40\left(\mathrm{~s}, 2 \mathrm{H},-\mathrm{OCH}_{2}\right), 7.00(\mathrm{~d}, 2 \mathrm{H}$, aromatic-H), $7.50(\mathrm{~m}, 2 \mathrm{H}$, aromatic- $\mathrm{H}), 7.80(\mathrm{~m}, 2 \mathrm{H}$, 
aromatic- $\mathrm{H}), 7.90(\mathrm{~d}, 2 \mathrm{H}$, aromatic- $\mathrm{H}), 8.00(\mathrm{~d}, 2 \mathrm{H}$, aromatic- $\mathrm{H}), 8.10(\mathrm{~s}, 1 \mathrm{H}$, aromatic- $\mathrm{H}), 8.30(\mathrm{~d}, 1 \mathrm{H}$, aromatic-H), $9.78(\mathrm{~s}, 1 \mathrm{H},-\mathrm{NH})$.ESI-MS: $m / z=464[\mathrm{M}+\mathrm{H}]$.

\section{2-(4-((1-(2-Fluorophenyl-1H-1,2,3-triazol-4-yl)methoxy)phenyl)quinazolin-4(3H)-one (7c)}

Yield: $87 \%$, m. p. $263-265{ }^{\circ} \mathrm{C}$. IR (KBr): v 3417, 3063, 2924, 1677, 1599, 1561, $1247 \mathrm{~cm}^{-1} .{ }^{1} \mathrm{H}$ NMR $\left(\mathrm{CDCl}_{3}\right): \delta 5.40\left(\mathrm{~s}, 2 \mathrm{H},-\mathrm{OCH}_{2}-\right), 7.08(\mathrm{~d}, 2 \mathrm{H}$, aromatic- $\mathrm{H}), 7.22-7.38(\mathrm{~m}, 3 \mathrm{H}$, aromatic- $\mathrm{H}), 7.40-7.47(\mathrm{~m}$, $2 \mathrm{H}$, aromatic-H), 7.76-7.80(m,3H,aromatic-H) 8.15-8.20 (m, 3H, aromatic-H), 11.20 (brs, 1H, -NH). ESIMS: $m / z=414[\mathrm{M}+\mathrm{H}]$.

\section{2-(4-((1-(4-chlorophenyl-1H-1,2,3-triazol-4-yl)methoxy) phenyl) quinazolin-4(3H)-one (7d)}

Yield: $92 \%$, m. p. $263-265{ }^{\circ} \mathrm{C}$. IR (KBr): v 3440, 3055, 1650, 1605, 1560, 1482, $1442 \mathrm{~cm}^{-1}$. ${ }^{1} \mathrm{H}$ NMR $\left(\mathrm{CDCl}_{3}\right): \delta 4.80\left(\mathrm{~s}, 2 \mathrm{H},-\mathrm{OCH}_{2}-\right), 7.00-7.05(\mathrm{~m}, 2 \mathrm{H}, \mathrm{Ar}-\mathrm{H}), 7.60-7.62(\mathrm{~m}, 3 \mathrm{H}$, aromatic- $\mathrm{H}), 8.00-8.05(\mathrm{~m}$, $2 \mathrm{H}$, aromatic-H), 8.10-8.21 (m, 6H, aromatic-H). ESI-MS: $m / z=430[\mathrm{M}+\mathrm{H}], 432[\mathrm{M}+2]$.

\section{2-(4-((1-(2,4-dichlorophenyl-1H-1,2,3-triazol-4-yl)methoxy) phenyl)quinazolin-4(3H)-one (7e)}

Yield: $92 \%$, m. p. $240-242{ }^{\circ} \mathrm{C}$. IR (KBr): v 3447, 3063, 2918, 2850, 1664, 1603, 1566, 1518, $1486 \mathrm{~cm}^{-1}$. ${ }^{1} \mathrm{H}$ NMR $\left(\mathrm{CDCl}_{3}\right): \delta 5.40\left(\mathrm{~s}, 2 \mathrm{H},-\mathrm{OCH}_{2}-\right), 7.20(\mathrm{~d}, 1 \mathrm{H}$, aromatic-H), $7.50(\mathrm{~s}, 1 \mathrm{H}$, aromatic- $\mathrm{H}), 7.65-7.85$ $(\mathrm{m}, 5 \mathrm{H}$, aromatic- $\mathrm{H}), 8.00-8.05(\mathrm{~m}, 2 \mathrm{H}$, aromatic- $\mathrm{H}), 8.20-8.38(\mathrm{~m}, 3 \mathrm{H}$, aromatic-H). ESI-MS: $\mathrm{m} / \mathrm{z}=464$ $[\mathrm{M}+\mathrm{H}], 466[\mathrm{M}+2], 468[\mathrm{M}+4]$.

\section{2-(4-((1-(2-Nitrophenyl-1H-1,2,3-triazol-4-yl)methoxy) phenyl)quinazolin-4(3H)-one (7f)}

Yield: $82 \%$, m. p. $226-228{ }^{\circ} \mathrm{C}$. IR ( $\left.\mathrm{KBr}\right): v 3444,2925,2859,1671,1462 \mathrm{~cm}^{-1} .{ }^{1} \mathrm{H}$ NMR $\left(\mathrm{CDCl}_{3}\right): \delta 5.41$ $\left(\mathrm{s}, 2 \mathrm{H},-\mathrm{OCH}_{2}-\right), 7.15(\mathrm{~s}, 1 \mathrm{H}, \mathrm{NH}), 7.80(\mathrm{~m}, 4 \mathrm{H}$, aromatic- $\mathrm{H}), 8.00(\mathrm{~d}, 1 \mathrm{H}$, aromatic-H), $8.18(\mathrm{~m}, 4 \mathrm{H}$, aromatic-H), $8.38(\mathrm{~d}, 2 \mathrm{H}$, aromatic-H). ESI-MS: $m / z=441[\mathrm{M}+\mathrm{H}]$.

\section{2-(4-((1-(4-Nitrophenyl-1H-1,2,3-triazol-4-yl)methoxy) phenyl)quinazolin-4(3H)-one (7g)}

Yield: $92 \%$, m. p. $233-235^{\circ} \mathrm{C}$. IR (KBr): v 3448, 3079, $1672,1603,1483 \mathrm{~cm}^{-1} .{ }^{1} \mathrm{H}$ NMR $\left(\mathrm{CDCl}_{3}\right): \delta 4.80$ $\left(\mathrm{s}, 2 \mathrm{H},-\mathrm{OCH}_{2}-\right), 7.15(\mathrm{~d}, 2 \mathrm{H}$, aromatic- $\mathrm{H}), 7.20(\mathrm{~d}, 2 \mathrm{H}$, aromatic- $\mathrm{H}), 7.58(\mathrm{~d}, 1 \mathrm{H}$, aromatic- $\mathrm{H}), 8.00(\mathrm{~d}$, $2 \mathrm{H}$, aromatic- $\mathrm{H}), 8.30(\mathrm{~d}, 4 \mathrm{H}$, aromatic- $\mathrm{H}), 8.48(\mathrm{~d}, 2 \mathrm{H}$, aromatic-H). ESI-MS: $m / z=441[\mathrm{M}+\mathrm{H}]$.

\section{2-(4-((1-(3-(Trifluomethyl)phenyl-1H-1,2,3-triazol-4-yl)methoxy)phenyl)quinazolin-4(3H)-one (7h)}

Yield: $89 \%$, m. p. $263-265^{\circ} \mathrm{C}$. IR (KBr): v 3447, 2918, 1664, 1518, $1243,765 \mathrm{~cm}^{-1} .{ }^{1} \mathrm{H} \mathrm{NMR}\left(\mathrm{CDCl}_{3}\right): \delta$ $5.40\left(\mathrm{~s}, 2 \mathrm{H},-\mathrm{OCH}_{2}-\right), 7.20(\mathrm{~d}, 1 \mathrm{H}$, aromatic- $\mathrm{H}), 7.50(\mathrm{~m}, 2 \mathrm{H}$, aromatic- $\mathrm{H}), 7.80-7.85(\mathrm{~m}, 4 \mathrm{H}$, aromatic$\mathrm{H}), 7.98(\mathrm{~d}, 2 \mathrm{H}$, aromatic- $\mathrm{H}), 8.08(\mathrm{~d}, 2 \mathrm{H}$, aromatic- $\mathrm{H}), 8.18(\mathrm{~s}, 1 \mathrm{H}$, aromatic- $\mathrm{H}), 8.30(\mathrm{~d}, 1 \mathrm{H}$, aromaticH), 9.80 (brs, $1 \mathrm{H},-\mathrm{NH})$. ESI-MS: $m / z=464[\mathrm{M}+\mathrm{H}]$.

\section{RESULTS AND DISCUSSION}

Because of several synthetic, biological, and pharmacological applications of quinazolinone derivatives, the present work is aimed to synthesize new triazole containing quinazolinone derivatives. On the other hand, in the search for new drugs, if any structural modification on existing quinazolinone that leads to possess significant biological activities. Therefore, there is an urgent need to develop synthetic methods for the quinazolinone skeleton and its analogs. In this context, synthesized new analogs of 1,2,3-triazole substituted quinazolinone from 4-hydroxy benzaldehyde, propoxyl bromide and anthranilamide.

4-Hydroxy benzaldehyde (1) was treated with propoxyl bromide (2) in presence of $\mathrm{K}_{2} \mathrm{CO}_{3}$ in dry $N, N^{\prime}-$ dimethyl formamide at RT for $6 \mathrm{~h}$ to get $O$-alkylated intermediate of 4-(prop-2-yn-1-yloxy)benzaldehyde (3), which upon treated with anthranilamide (4) in presence of iodine in dimethyl sulfoxide at $100{ }^{\circ} \mathrm{C}$ for $3 \mathrm{~h}$ obtained an intermediate of 4-(prop-2-yn-1-yloxy)phenyl) quinazoline-4(3H)-one (5) in excellent yield $(92 \%)$. Finally, the desired triazole products $7 \mathbf{a}-\mathbf{h}$ have been achieved by the reaction of the intermediate of 4-(prop-2-yn-1-yloxy)phenyl) quinazoline-4(3H)-one (5) with various phenylazides (6ah) in presence of copper sulphate and $\mathrm{Na}+$ ascorbate in 1:1 ratio of THF-water at RT for $3 \mathrm{~h}$ (Scheme-1). Newly synthesized derivatives were confirmed by using ${ }^{1} \mathrm{H}$ NMR, IR and ESI-Mass spectral analysis and are in good agreement with the proposed derivative structures. In ${ }^{1} \mathrm{H}$ NMR the triazole characteristic 
RASĀYAN J. Chem.

Vol. 14 | No. 3 |1534-1538| July - September | 2021

signal appeared in the range of $\delta 7.50-7.62 \mathrm{ppm}$ of $7 \mathbf{a}$ as a singlet and triazole attached of $-\mathrm{OCH}_{2}$ - protons detected as single at $\delta 5.39 \mathrm{ppm}$. The derivative structures were confirmed further by ESI-MS data which molecular ion peak was displayed at $m / z=396[\mathrm{M}+\mathrm{H}]^{+}$of $\mathrm{C}_{22} \mathrm{H}_{17} \mathrm{~N}_{5} \mathrm{O}_{2}$ and IR spectra shown absorption bands at 1602-1664 $\mathrm{cm}^{-1}$ and $1520,1482,1444 \mathrm{~cm}^{-1}$ which corresponded to $\mathrm{C}=\mathrm{O},-\mathrm{OCH} 2, \mathrm{~N}-\mathrm{N}, \mathrm{N}=\mathrm{N}$ as characteristics.<smiles>O=c1[nH]c(-c2ccc(COc3cn(-c4ccccc4)nn3)cc2)nc2ccccc12</smiles>

$7 a$<smiles>O=c1[nH]c(-c2ccc(OCc3cn(-c4ccccc4C(F)(F)F)nn3)cc2)nc2ccccc12</smiles><smiles>O=c1[nH]c(-c2ccc(OCc3cn(-c4ccccc4F)nn3)cc2)nc2ccccc12</smiles><smiles>O=c1[nH]c(-c2ccc(OCc3cn(-c4ccc(Cl)cc4)nn3)cc2)nc2ccccc12</smiles><smiles>O=c1[nH]c(-c2ccc(Cc3cn(-c4ccc(Cl)cc4Cl)nn3)cc2)nc2ccccc12</smiles><smiles>O=c1[nH]c(-c2ccc(OCc3cn(-c4ccccc4[N+](=O)[O-])nn3)cc2)nc2ccccc12</smiles><smiles>O=c1[nH]c(-c2ccc(OCc3cn(-c4ccc([N+](=O)[O-])cc4)nn3)cc2)nc2ccccc12</smiles><smiles>O=c1[nH]c(-c2ccc(OCc3cn(-c4cccc(C(F)(F)F)c4)nn3)cc2)nc2ccccc12</smiles>

Fig.-1

\section{CONCLUSION}

In conclusion, we have designed and synthesized a new analog of 1,2,3-triazole substituted quinazolinone from 4-hydroxy benzaldehyde, propoxyl bromide and anthranilamide in good to excellent yields. Newly synthesized derivatives were confirmed by using ${ }^{1} \mathrm{H}$ NMR, IR and ESI-Mass spectral analysis and agreement with the proposed derivatives.

\section{REFERENCES}

1. L. A. Skelton, M. G. Ormerod, J. Titley, R. Kimbell, L. A. Brunton, A. L. Jackman, British Journal of Cancer, 79, 1692(1999), https://doi.org/10.1038/sj.bjc.6690270 .

2. Yi Xia, Zheng-Yu Yang, Mann-Jen Hour, Sheng-Chu Kuo, Peng Xia, Kenneth F. Bastow, Yukuna Nakanishi, Priya Nampoothiri, Torben Hackl, Ernest Hamel, Kuo-Hsiung Lee, Bioorganic Medicinal Chemistry Letters, 11, 1193(2001).

3. V. Alagarswamy, R. Revathi, S. Meena, K. V. Ramaseshu, S. Rajasekaran, E. De Clercq, Indian Journal of Pharmaceutical Science, 4, 459(2004).

4. Maher F. El-Zohry, Abd El-Hamed N. Ahmed, Farghaly A. Omar, Mohamed A. Abd-Alla, Journal of Chemical Technology \& Biotechnology, 53, 329(1992), https://doi.org/10.1002/jctb.280530403

5. David J. Connolly, Declan Cusack, Timothy P. O'Sullivan, Patrick J. Guiry, Tetrahedron, 61, 10153(2005), https://doi.org/0.1016/j.tet.2005.07.010

6. R. Rohini, K. Shanker, P. Muralidhar Reddy, V. Chandra Sekhar, V. Ravinder, Arch Pharma Chemical Life Sciences, 342, 533(2009), https://doi.org/10.1002/ardp.200900068 
RASĀYAN J. Chem.

Vol. 14 | No. 3 |1534-1538| July - September | 2021

7. Zhong-Ze Ma, Yoshio Hano, Taro Nomura, Ying-Jie Chen, Heterocycles, 46, 541(1997), https://doi.org/10.3987/COM-97-S65

8. M. E. Wall, M. C. Qani, C. E. Cook, K. H. Palmer, A. T. Mcphail, G. A. Sim, Journal of American Chemical Society, 88, 3888(1966), https://doi.org/10.1021/ja00968a057

9. C. H. Takimoto, J. Wright, S. G. Arbuck, Biochimica Biophysica Acta, 1400, 107(1998), https://doi.org/10.1016/s0167-4781(98)00130-4

10. J. O'Leary, F. M. Muggia, European Journal of Cancer, 34, 1500(1998), https://doi.org/10.1016/S0959-8049(98)00229-9

11. L. B. Saltz, J. V. Cox, C. Blanke, L. S. Rosen, L. Fechrenbacher, M. J. Moore, J. A. Maroun, S. P. Ackland, P. K. Locker, N. Pirotta, G. L. Elfring, L. L. Miller, The New England Journal of Medicine, 343, 905(2000), https://doi.org/10.1056/NEJM200009283431302

12. R. T. Penson, K. Kronish, Z. Duan, A. J. Feller, P. Stark, S. E. Cook, L. R. Duska, A. F. Fuller, A. K. Goodman, N. Nikrui, K. M. MacNeill, U. A. Matulonis, F. I. Preffer, M. V. Seiden, International Journal of Gynecological Cancer, 10, 33(2000), https://doi.org/10.1046/j.1525-1438.2000.00003.x

13. U. Vanhoefer, A. Harstrick, W. Achterrath, S. Cao, S. Seeber, Y. M. Rustum, Journal of Clinical Oncology, 19, 1501(2001), https://doi.org/10.1200/jco.2002.20.5.1425

14. H. Ulukan, P. W. Swaan, Drugs, 62, 2039(2002), https://doi.org/10.2165/00003495-20026214000004

15. R. Garcia-Carbenero, J. G. Supko, Clinical Cancer Research, 8, 641(2002), https://doi.org/10.1158/1078-0432.CCR-06-1347

[RJC-6259/2020] 